\title{
Effect of psychosocial interventions on social functioning in depression and schizophrenia: meta-analysis
}

\author{
Mary J. De Silva, Sara Cooper, Henry Lishi Li, Crick Lund and Vikram Patel
}

\section{Background}

Psychosocial interventions may contribute to reducing the burden of mental disorders in low- and middle-income (LAMI) countries by improving social functioning, but the evidence has not been systematically reviewed.

\begin{abstract}
Aims
Systematic review and meta-analysis of the effect of psychosocial interventions on social functioning in people with depression and schizophrenia in LAMI countries.
\end{abstract}

\section{Method}

Studies were identified through database searching up to March 2011. Randomised controlled trials were included if they compared the intervention group with a control group receiving placebo or treatment as usual. Random effects meta-analyses were performed separately for depressive disorders and schizophrenia and for each intervention type.

\section{Results}

Of the studies that met the inclusion criteria $(n=24), 21$ had sufficient data to include in the meta-analysis. Eleven depression trials showed good evidence for a moderate positive effect of psychosocial interventions on social functioning (standardised mean difference $(S M D)=0.46,95 \%$ $\mathrm{Cl} 0.24-0.69, n=4009$ ) and ten schizophrenia trials showed a large positive effect on social functioning $(S M D=0.84$, 95\% Cl 0.49-1.19, $n=1671$ ), although seven of these trials were of low quality. Excluding these did not substantially affect the size or direction of effect, although the precision of the estimate was substantially reduced $(\mathrm{SMD}=0.89$, $95 \% \mathrm{Cl} \mathrm{0.05-1.72,} n=863$ ).

\section{Conclusions}

Psychosocial interventions delivered in out-patient and primary care settings are effective at improving social functioning in people with depression and should be incorporated into efforts to scale up services. For schizophrenia there is an absence of evidence from high-quality trials and the generalisabilty of the findings is limited by the over-representation of trials conducted in populations of hospital patients in China. More high-quality trials of psychosocial interventions for schizophrenia delivered in out-patient settings are needed.

\section{Declaration of interest}

$$
\text { None. }
$$

Depression and schizophrenia cause severe impairments in social functioning and rank among the leading mental health causes of the global burden of disease. ${ }^{1,2}$ Impairment of social functioning, defined as 'an individual's ability to perform and fulfil normal social roles, ${ }^{3}$ is a major reason for the high levels of stigma and disability associated with these mental disorders. Although there have been numerous reviews of the clinical effectiveness of interventions for mental disorders, ${ }^{4-7}$ the effect of psychosocial interventions on social functioning has not been reviewed. Only 17 of the 62 Cochrane reviews of psychosocial interventions to treat depression or schizophrenia include social functioning outcomes, with only a tiny fraction of the included trials from low- and middle-income (LAMI) countries (the results of these reviews are summarised in online Table DS1). This review aims to synthesise all randomised controlled trials (RCTs) conducted in LAMI countries evaluating the effectiveness of psychosocial interventions to treat depression or schizophrenia on social functioning outcomes.

\section{Method}

The methods and results in this paper are presented according to the PRISMA statement for reporting systematic reviews. ${ }^{8}$

\section{Selection of studies}

A protocol for the review was developed in collaboration with a Cochrane information scientist. The Appendix lists the inclusion and exclusion criteria for the review. In summary, the review included all RCTs that assessed the effect of psychosocial interventions on the social functioning of people living with depressive disorders or schizophrenia in LAMI countries. Psychosocial interventions were defined broadly as any nonpharmacological or physical intervention, ${ }^{9}$ and comprised structured psychotherapies such as cognitive-behavioural therapy (CBT); psychosocial interventions such as social skills training; alternative therapies including exercise and art therapy; and collaborative care stepped-care interventions that combine a series of different interventions. Trials were included as long as they compared the intervention group with a control group receiving a placebo or treatment as usual (TAU). This ensured that the effectiveness of the intervention was assessed, rather than its equivalence to a similar treatment.

To be included, trials must have quantitatively assessed the effect of the intervention on patient social functioning, measured using a validated tool. Social functioning can be seen as one aspect of disability comprising social and physical functioning, both subdomains of quality of life. ${ }^{10}$ Core domains include: occupation, education, household role, marital functioning, parental role, leisure and recreational activities and self-care, ${ }^{11}$ as well as an individuals' satisfaction with their ability to meet these roles. ${ }^{12}$ Because social functioning is a subdomain of quality of life, ${ }^{10}$ quality of life measures were excluded from the review. Equally, scales reporting general health status (such as the Short Form 36-item questionnaire (SF-36) $)^{13}$ ) were excluded, although studies that reported the results of the social functioning subscale of general health scales were included. Where studies included more than one measure of social functioning, results for the scale 
that captured the most domains of social functioning were extracted.

The search was not restricted by date, language or publication status. The following electronic databases were searched: Medline, PsycINFO, Cochrane Central, Econlit and ISI Web of Science using Medical Subject Heading (MeSH) terms (or equivalent terms) for published peer-reviewed journal articles. The online supplement lists the full search strategy. Randomised controlled trials for all mental disorders were searched, and those relating to depression and schizophrenia manually selected. The last search was conducted in March 2011. The reference lists of all selected papers were screened and authors of relevant studies contacted to seek additional studies and request information not present in the published paper.

Initial screening of irrelevant abstracts involved one author (M.J.D.S.) searching through the database of search results for papers that were not related to mental health. Two authors (M.J.D.S. and S.C.) then independently screened the titles and abstracts of the remaining search results and the full text copies of all potentially relevant studies to determine whether they met the pre-specified inclusion criteria. Disagreements were resolved by discussion among all authors.

Data were extracted by two authors using a standard data extraction form including inclusion criteria for participants, intervention and control groups, outcome measures and effect estimates. The quality of included studies was assessed using the Cochrane risk of bias tool ${ }^{14}$ by two authors. Risk of bias was assessed both at the study level (for example sequence generation and allocation concealment) and at the outcome level (for example losses to follow-up for the social functioning outcome). Data for the meta-analyses were extracted by H.L.L. and double checked by M.J.D.S. Where trials reported more than one follow-up time point, data were extracted from the closest time point to 6 months for depression trials and 12 months for schizophrenia trials. These time points were chosen to reflect the longer-term effect that psychosocial interventions are anticipated to have on social functioning outcomes.

\section{Data analysis}

Statistical analyses were performed using Review Manager 5 for Windows 7. The post-treatment mean and standard deviation (s.d.) of the social functioning score in the intervention and control group were extracted along with the sample size in each group to calculate the standardised mean difference (SMD) for each trial to enable different outcome scales to be pooled. Where cluster RCTs were included, the mean post-treatment scores calculated from an appropriate analysis adjusted for clustering were used to enable them to be combined with the results of individually randomised trials. ${ }^{15}$ To correct for differences in the direction of the scales (for example some scales increase with increasing severity and others decrease), the mean values from one set of studies was multiplied by -1 to ensure that all the scales point in the same direction. Acknowledging the heterogeneity in interventions and study design, random effects meta-analyses were performed separately for depressive disorders and schizophrenia and within this separately for each intervention type. The $I^{2}$ statistic was used to assess heterogeneity between trials.

A number of sensitivity analyses were conducted. To control for study quality, trials that had a risk of bias for allocation concealment, or for whom allocation concealment could not be assessed but who had a risk of bias for sequence generation and/ or masking of outcome assessment, were excluded from the meta-analysis. Separate meta-analyses were conducted to assess the long- and short-term effects on social functioning. Short-term follow-up was defined as less than 6 months for depressive disorders and less than 12 months for schizophrenia, and longterm follow-up as more than 6 months for depression and more than 12 months for schizophrenia. We contacted the authors for missing data necessary for the meta-analysis. Where these data were not available we conducted a sensitivity analysis to exclude those studies with a high risk of bias, including those with bias due to missing data for their outcome assessment. We did not impute missing data as we were unable to obtain the raw data from authors. Lastly, funnel plots for the primary meta-analyses were generated to assess possible publication bias.

\section{Results}

Figure 1 presents the search and selection process for the review. A total of 9592 unique records were obtained, of which 24 trials met the inclusion criteria. Thirteen papers were in English, ten in Chinese and one in Spanish.

\section{Measurement of social functioning}

Online Table DS2 lists the social functioning tools used by the included trials. The 24 included trials used 10 different scales to measure social functioning, confirming the previously reported

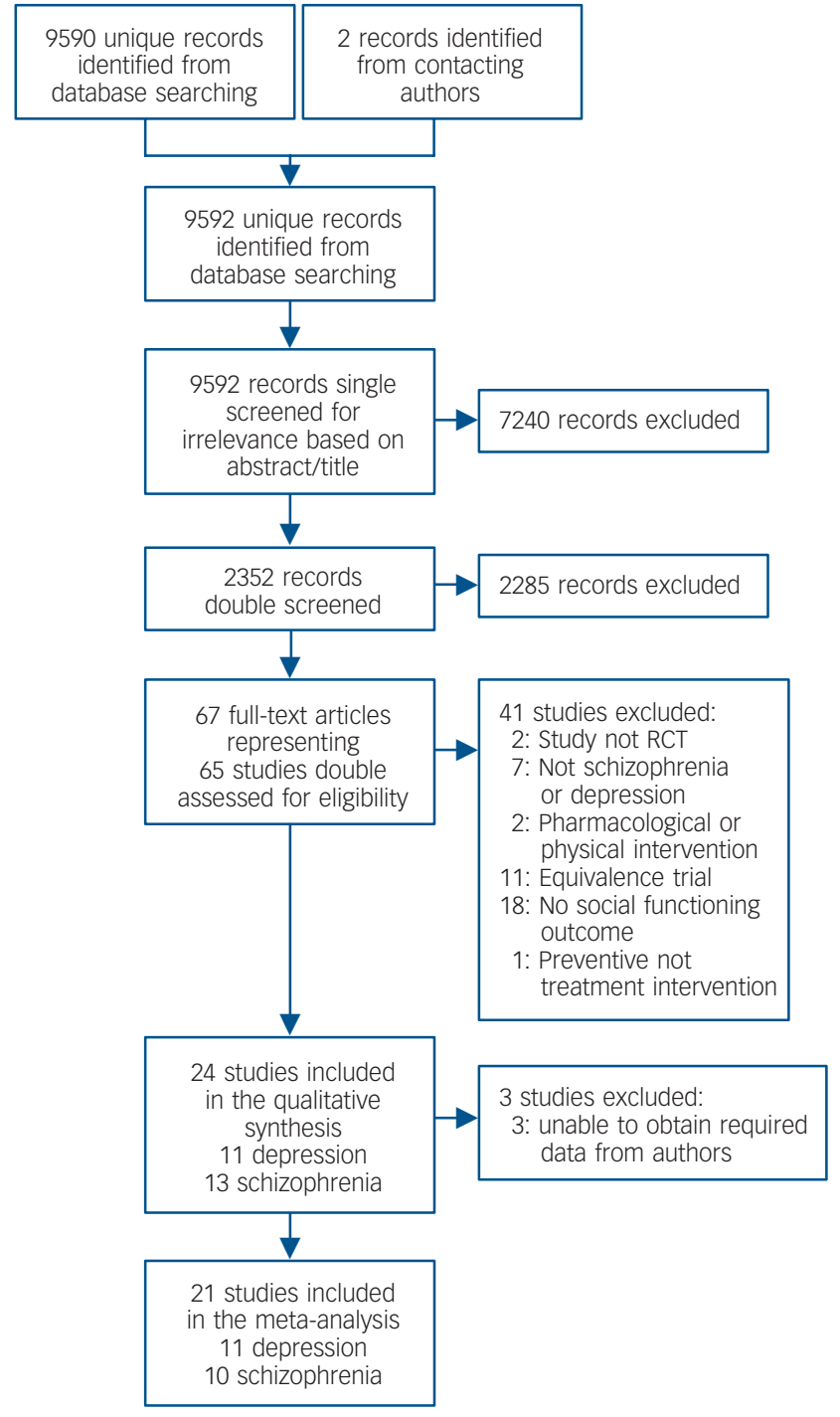

Fig. 1 Selection of studies 
lack of consensus on its measurement. ${ }^{16}$ Seven of the included tools were patient self-assessments, and three were clinician-rated. Half were developed to measure social functioning in a psychiatric population, and four specifically for populations in LAMI countries. Many of the tools were sophisticated in their measurement of a number of domains of social functioning, although no tool measured all domains, and had been appropriately validated in either a number of populations, or specifically in the population in which they were used. Table 1 summarises the trials included in the review separately for depression and schizophrenia.

\section{Effect of psychosocial interventions to treat depression}

In total 11 trials assessed the effect of interventions to treat depression, 6 assessed multicomponent collaborative care interventions, three interpersonal therapy (IPT), 1 problem-solving therapy, and 1 Morita therapy. Four of the trials were from Chile, three from China, and the remainder from India, Brazil and Uganda. The majority of trials were set in out-patient, primary care or community settings. Five of the trials used non-mental health specialists to deliver the intervention through task-sharing. Only one trial was assessed as having an overall risk of bias and nine had long-term follow-up of more than 6 months. Figure 2 presents the forest plot for the main results meta-analysis with follow-up clustered around 6 months. Online Table DS3 reports the characteristics and main findings of the depression trials and online Figs DS1-3 presents the forest plots for the sensitivity analyses.

All 11 depression trials were suitable for inclusion in the metaanalysis. The combined SMD for all interventions was 0.46 (95\% CI $0.24-0.69, P \leqslant 0.001, I^{2}=90 \%, n=4009$ ), indicating small to moderate improvements in social functioning based on the rule of thumb interpretation of SMDs whereby 0.2 represents a small effect, 0.5 a moderate effect and 0.8 a large effect. ${ }^{29}$ Excluding the one trial with a risk of bias did not affect this conclusion, and the magnitude of effect was the same for both short- and long-term follow-up.

There was robust evidence from the six trials evaluating multicomponent interventions for a small improvement in social functioning (SMD $=0.35,95 \%$ CI $0.11-0.59, P \leqslant 0.001, I^{2}=89 \%$, $n=3291)$. These multicomponent interventions involved structured pharmacotherapy, psychoeducation, adherence support and in some cases IPT or cognitive trauma-based therapy. These interventions were often delivered by non-specialist health workers as part of a multidisciplinary team in a stepped-care model. The control arm received TAU, which frequently included access to pharmacotherapy or psychological therapy if indicated.

There was evidence from three trials of a large, positive impact of IPT on social functioning ( $\mathrm{SMD}=0.84,95 \%$ CI $0.40-1.29$, $P=0.0002, I^{2}=67 \%, n=360$ ). Two trials examined the effect of group IPT delivered in $12^{20}$ or $16^{18}$ sessions and the third assessed the impact of 16 sessions of individual IPT. ${ }^{19}$ There was not enough evidence to assess the effect of problem-solving therapy or Morita therapy as only one trial respectively assessed these interventions.

\section{Effect of psychosocial interventions to treat schizophrenia}

Thirteen trials assessed the effect of interventions to treat schizophrenia: 3 trials assessed the effect of family psychoeducation, 1 patient psychoeducation, 1 social skills training, 1 art therapy, 4 multicomponent structured psychotherapies and 3 communitybased care interventions. In contrast to the depression trials, most $(11 / 13)$ were conducted in China in hospital in-patient populations and only two used non-specialists to deliver the intervention. No trials were included from Sub-Saharan Africa or South Asia. Seven were assessed as having a risk of bias and five had strict inclusion criteria limiting the generalisability of the results. Three trials did not contain sufficient data to be included in the meta-analysis, ${ }^{30-32}$ and as we were unable to obtain this information from the authors, these trials are included in the

\begin{tabular}{|c|c|c|c|}
\hline & $\begin{array}{l}\text { Depression studies, } n \\
(n=11)\end{array}$ & $\begin{array}{l}\text { Schizophrenia studies, } n \\
\qquad(n=13)\end{array}$ & Total, $n$ \\
\hline \multicolumn{4}{|l|}{ Country } \\
\hline Chile & 4 & 0 & 4 \\
\hline Brazil & 1 & 1 & 2 \\
\hline China & 3 & 11 & 14 \\
\hline India & 2 & 0 & 2 \\
\hline Uganda & 1 & 0 & 1 \\
\hline Turkey & 0 & 1 & 1 \\
\hline \multicolumn{4}{|l|}{ Setting } \\
\hline Hospital in-patient & 2 & 7 & 9 \\
\hline Hospital out-patient & 4 & 4 & 8 \\
\hline Primary healthcare & 4 & 0 & 4 \\
\hline Community & 1 & 2 & 3 \\
\hline \multicolumn{4}{|l|}{ Intervention } \\
\hline Psychological therapy & 4 & 9 & 13 \\
\hline Other intervention ${ }^{a}$ & 1 & 1 & 2 \\
\hline Multicomponent collaborative care & 6 & 3 & 9 \\
\hline Intervention delivered by non-mental health specialist & 5 & 2 & 7 \\
\hline \multicolumn{4}{|l|}{ Study design } \\
\hline Long-term follow-up ${ }^{\mathrm{b}}$ & 9 & 6 & 15 \\
\hline Strict inclusion criteria & 4 & 5 & 9 \\
\hline Small sample size (<50 participants per arm) & 3 & 8 & 11 \\
\hline Assessed as overall high risk of bias & 1 & 7 & 8 \\
\hline
\end{tabular}




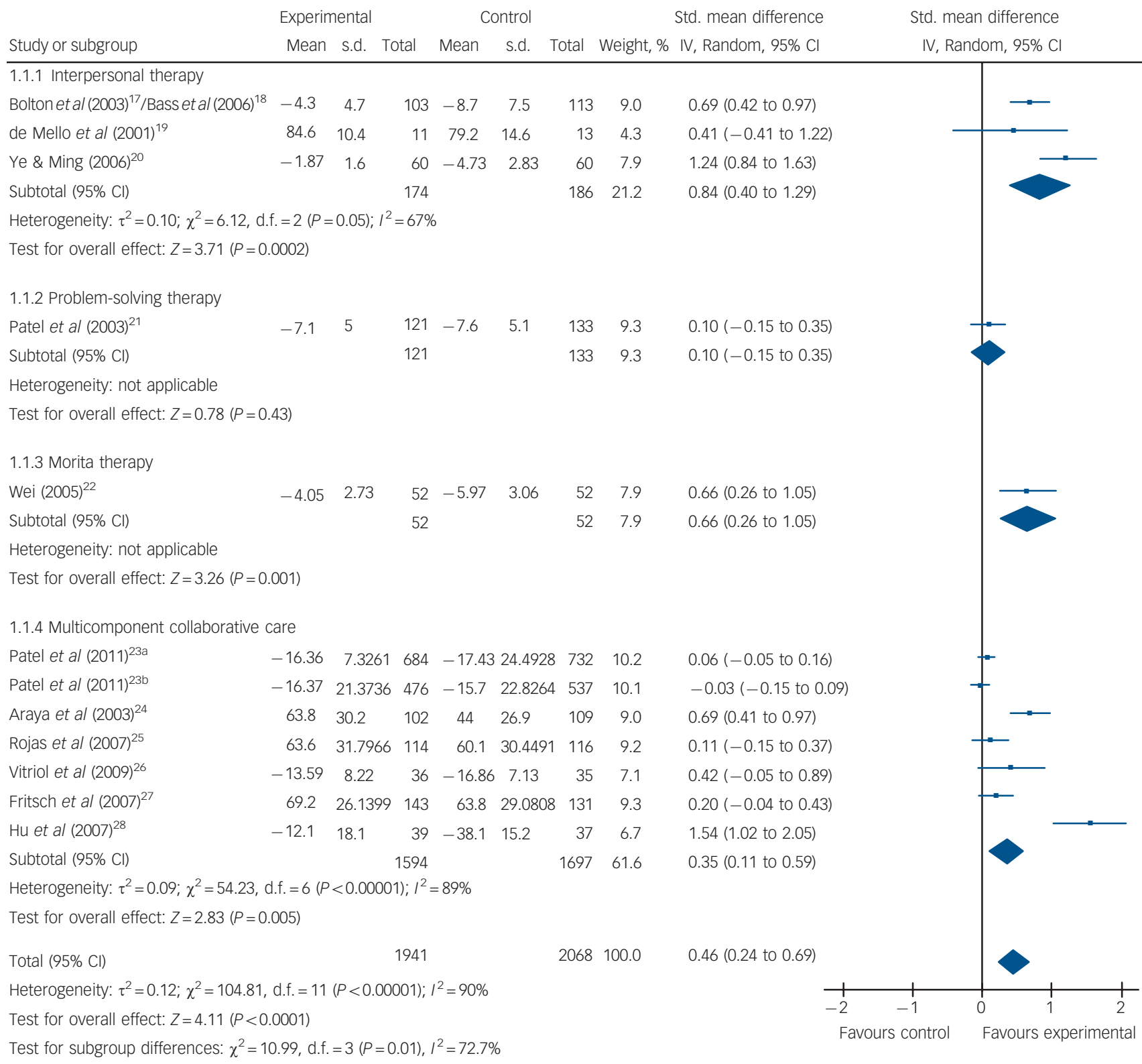

Fig. 2 Depression: all studies (6-month follow-up).

Patel et al (2011): a. recruited from public primary healthcare clinics, b. Recruited from private general practice clinics.

qualitative synthesis of results only. Figure 3 presents the forest plot for the main results meta-analysis with follow-up clustered around 12 months. Online Table DS4 reports the characteristics and main findings of the schizophrenia trials. Online Figs DS4-6 presents the forest plots for the sensitivity analysis.

The combined SMD for all interventions was 0.84 (95\% CI $\left.0.49-1.19, \quad P \leqslant 0.001, \quad I^{2}=89 \%, \quad n=1671\right), \quad$ indicating large improvements in social functioning. Excluding the seven trials with a risk of bias did not substantially affect the size or direction of effect, although the precision of the estimate was substantially reduced due to the smaller pooled sample sizes $(\mathrm{SMD}=0.89$, $95 \%$ CI $\left.0.05-1.72, P \leqslant 0.001, I^{2}=91 \%, n=863\right)$. The effect of the interventions on social functioning increased over time, with moderate effect sizes at less than 12 months' follow-up $\left(\mathrm{SMD}=0.71,95 \%\right.$ CI $\left.0.36-1.06, P \leqslant 0.001, I^{2}=88 \%, n=1718\right)$, increasing to a large effect on social functioning at more than 12 months' follow-up ( $\mathrm{SMD}=0.93,95 \%$ CI $0.37-1.49, P \leqslant 0.001$, $\left.I^{2}=95 \%, n=1409\right)$. However, the risk of bias associated with seven of these ten studies limits the strength of the evidence from this meta-analysis.

There was good evidence from four trials of large improvements in social functioning due to multicomponent structured psychotherapies against TAU with both groups receiving anti-

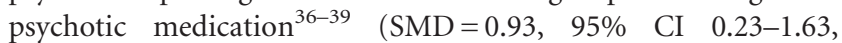
$P \leqslant 0.0001, I^{2}=89 \%, n=893$ ). All trials included psychoeducation supplemented with at least two additional therapies comprising skills training, CBT, IPT and family therapy. Three of these trials had a low risk of bias and a sensitivity analysis restricted to these trials did not affect this finding.

There was weak evidence from three poor-quality trials of a large positive effect of psychoeducation on social functioning $\left(\mathrm{SMD}=1.15,95 \%\right.$ CI $\left.0.06-2.25, P \leqslant 0.001, I^{2}=95 \%, n=362\right)$. Two of these trials assessed the impact of family psychoeducation, $^{33,34}$ and one patient psychoeducation ${ }^{35}$ compared with TAU, with both groups receiving antipsychotic medication. The meta-analysis was skewed by the study of individual patient 


\begin{tabular}{|c|c|c|c|c|c|c|c|c|c|c|c|}
\hline \multirow[b]{2}{*}{ Study or subgroup } & \multicolumn{3}{|c|}{ Experimental } & \multicolumn{3}{|c|}{ Control } & \multicolumn{2}{|r|}{ Std. mean difference } & \multicolumn{3}{|c|}{ Std. mean difference } \\
\hline & Mean & s.d. & Total & Mean & s.d. & Total & Weight, \% & IV, Random, 95\% Cl & \multicolumn{3}{|c|}{ IV, Random, 95\% Cl } \\
\hline \multicolumn{12}{|l|}{ 2.1.1 Psychoeducation } \\
\hline Li \& Arthur $(2005)^{33}$ & 78 & 10.3 & 36 & 70.2 & 15.9 & 33 & 9.8 & $0.58(0.10$ to 1.06$)$ & & & \\
\hline Wang et al (2008) ${ }^{34}$ & -4.2 & 0.9 & 98 & -4.7 & 1 & 95 & 11.2 & 0.52 (0.24 to 0.81$)$ & & & \\
\hline Wei et al (1997) ${ }^{35}$ & -0.8 & 0.3 & 50 & -2.1 & 0.7 & 50 & 9.6 & $2.40(1.88$ to 2.91$)$ & & & $\longrightarrow$ \\
\hline Subtotal $(95 \% \mathrm{Cl})$ & & & 184 & & & 178 & 30.6 & 1.15 (0.06 to 2.25$)$ & & & \\
\hline \multicolumn{12}{|c|}{$\begin{array}{l}\text { Heterogeneity: } \tau^{2}=0.89 ; \chi^{2}=40.25 \text {, d.f. }=2(P<0.00001) ; I^{2}=95 \% \\
\text { Test for overall effect: } Z=2.07(P=0.04)\end{array}$} \\
\hline \multicolumn{12}{|c|}{ 2.1.2 Multicomponent structured psychotherapies } \\
\hline Chen et al (2003) $)^{36}$ & -4.04 & 3.89 & 32 & -7.63 & 4.27 & 31 & 9.6 & 0.87 (0.35 to 1.39$)$ & & & \\
\hline Guo et al $(2010)^{37}$ & 82.9 & 8.1998 & 406 & 80.8 & 9.3465 & 5338 & 11.8 & $0.24(0.10$ to 0.38$)$ & & $\rightarrow$ & \\
\hline Yildiz et al (2004) ${ }^{38}$ & 132.6 & 33.85 & 15 & 96.2 & 30.24 & 15 & 7.6 & $1.10(0.33$ to 1.88$)$ & & & \\
\hline Zimmer et al $(2007)^{39}$ & 43.25 & 6.54 & 20 & 34.14 & 4.53 & 36 & 8.7 & 1.69 (1.05 to 2.32$)$ & & & \\
\hline Subtotal $(95 \% \mathrm{Cl})$ & & & 473 & & & 420 & 37.6 & $0.93(0.23$ to 1.63$)$ & & & \\
\hline \multicolumn{12}{|c|}{$\begin{array}{l}\text { Heterogeneity: } \tau^{2}=0.43 ; \chi^{2}=26.59 \text {, d.f. }=3(P<0.00001) ; I^{2}=89 \% \\
\text { Test for overall effect: } Z=2.60(P=0.009)\end{array}$} \\
\hline \multicolumn{12}{|l|}{ 2.1.3 Art therapy } \\
\hline Meng et al $(2005)^{40}$ & 67.69 & 15.03 & 50 & 56.93 & 15.24 & 50 & 10.4 & $0.71(0.31$ to 1.12$)$ & & & \\
\hline Subtotal $(95 \% \mathrm{Cl})$ & & & 50 & & & 50 & 10.4 & $0.71(0.31$ to 1.12$)$ & & & \\
\hline \multicolumn{12}{|c|}{ Heterogeneity: not applicable } \\
\hline \multicolumn{12}{|c|}{ Test for overall effect: $Z=3.45(P=0.0006)$} \\
\hline \multicolumn{12}{|c|}{ 2.1.4 Multicomponent community care } \\
\hline Li et al (2002) $)^{41}$ & -1.61 & 4.56 & 38 & -3.64 & 4.05 & 38 & 10.0 & 0.47 (0.01 to 0.92$)$ & & & \\
\hline Pang et al (2002) ${ }^{42}$ & -37 & 0.68 & 120 & -1.6 & 0.92 & 120 & 11.4 & $0.28(0.03$ to 0.54$)$ & & - & \\
\hline Subtotal $(95 \% \mathrm{Cl})$ & & & 158 & & & 158 & 21.4 & $0.33(0.10$ to 0.55$)$ & & & \\
\hline \multicolumn{12}{|c|}{$\begin{array}{l}\text { Heterogeneity: } \tau^{2}=0.00 ; \chi^{2}=0.47, \text { d.f. }=1(P<0.49) ; I^{2}=0 \% \\
\text { Test for overall effect: } Z=2.88(P=0.004)\end{array}$} \\
\hline \multirow{2}{*}{\multicolumn{9}{|c|}{$\begin{array}{l}\text { Heterogeneity: } \tau^{2}=0.27 ; \chi^{2}=85.38, \text { d.f. }=9(P<0.00001) ; I^{2}=89 \% \\
\text { Test for overall effect: } Z=4.68(P<0.00001)\end{array}$}} & & & \\
\hline & & & & & & & & & $\begin{array}{lll}-2 & -1 & 0\end{array}$ & 1 & 2 \\
\hline Test for subgroup diffe & $\chi^{2}=6.1$ & 13 , d.f. $=$ & $=3(P=0$ & .11), $1^{2}=$ & $51.1 \%$ & & & & Favours control & Favours & \\
\hline
\end{tabular}

Fig. 3 Schizophrenia: main results (12-month follow-up).

psychoeducation, which had a much larger effect than the two family interventions. A fourth trial on family psychoeducation that could not be included in the meta-analysis also reported a significant positive effect on social functioning. ${ }^{30}$ As four trials were assessed as having a high overall risk of bias (two because the risk of bias was unknown due to lack of information in the published paper), the level of evidence for psychoeducation is currently weak.

There was weak evidence from two trials with a high risk of bias $^{41,42}$ of a small increase in social functioning as a result of community-based interventions ( $\mathrm{SMD}=0.33,95 \%$ CI $0.10-0.55$, $P=0.004, I^{2}=88 \%, n=316$ ), despite neither trial showing an effect on clinical outcomes. The trials compared a package of interventions combining psychotherapies (mainly psychoeducation and family therapy) compared with either in-patient treatment or standard out-patient treatment, with both groups receiving medication. This finding was replicated in a third trial that could not be included in the meta-analysis because of a lack of data, which also showed a significant improvement in social functioning at 12 - and 18-month follow-up. ${ }^{32}$

There was not enough evidence to assess the effect of art therapy or social skills training as only one study respectively assessed these interventions.

\section{Publication bias}

Online Figs DS7 and 8 present the funnel plots to assess potential publication bias for the primary meta-analyses. We performed a visual inspection of the plots in line with recommendations not to perform statistical tests of asymmetry where there are a small number of trials or there is significant heterogeneity between trials. ${ }^{43}$ Although a visual inspection of the plots shows them to be somewhat asymmetrical, this asymmetry may have been caused by heterogeneity in intervention type rather than publication bias, as indicated by the large $I^{2}$ for the combined effect estimates. Some asymmetry may also have been caused by the tendency in this review for the poorer quality trials to show larger effects, as documented elsewhere. ${ }^{44}$

\section{Discussion}

\section{Main findings}

A total of 11 depression trials from 5 countries and 13 schizophrenia trials from 3 countries were included in this review. Overall, the results show that different types of psychosocial interventions are effective at improving social functioning in people with depression and schizophrenia in LAMI countries. For depression, there is strong evidence that stepped collaborative care interventions, often delivered by non-specialists and comprising structured pharmacotherapy, psychoeducation, adherence support and in some cases structured psychotherapy have moderate effects on improving patient social functioning up to 12 months from start of treatment. There was also some evidence that IPT, often delivered by non-specialists, is effective at improving social functioning over a 12-month period. For schizophrenia, interventions demonstrated a strong effect, but the interpretation of these findings is tempered by the risk of bias associated with seven of the ten trials. The generalisability of these findings is also restricted by the predominance of trials of hospital in-patients 
in China. However, there was good evidence from three high-quality trials that a combination of structured psychological therapies (for example psychoeducation, social skills training and IPT), delivered in combination with antipsychotic medication, leads to large improvements in patient social functioning compared with medication alone.

A striking finding of this review is that improvements in social functioning were maintained at long follow-up periods of over a year. In contrast to clinical improvements that are often observed early in the intervention, improvements in social functioning were sometimes only evident at later stages (for example Li \& Arthur $^{33}$ and Pang $e a^{42}$ ). It is likely that improvements in social functioning happen more slowly and subsequently to clinical improvements and that patients who recover symptomatically can be expected to experience a positive change in social functioning. Indeed, in the vast majority of included trials, concurrent improvements in both clinical and social functioning were observed. However, an intervention that improves social functioning may not necessarily have an impact on clinical symptoms: notably, the two trials assessing community care for schizophrenia demonstrated an impact on social functioning even though the intervention had no impact on clinical outcomes. ${ }^{41,42}$ This may be because these interventions involved shifting the locus of care to the community to promote re-integration following a hospital admission, rather than specific treatments for clinical symptoms. Increased efforts are needed to disentangle those aspects of interventions that are effective at improving clinical symptoms and social functioning, in order to ensure they are both cost-effective and acceptable to patients and care providers.

\section{Methodological limitations}

We note some of the limitations of the evidence included in this review that affect the strength of conclusions and generalisability of the results, particularly to efforts to scale up services for people with mental disorders in LAMI countries. ${ }^{45}$ For schizophrenia there was an absence of evidence from high-quality trials and the generalisabilty of the findings is limited by the over-representation of trials from China conducted in populations of hospital patients. Trials of task-shifted psychosocial interventions delivered in primary care are urgently needed. In contrast, all but one of the trials included in the depression meta-analysis were methodologically strong.

Additional limitations of the evidence included in this review include the short follow-up in a third of trials, potentially not allowing sufficient time to detect improvements in functioning in the intervention group. Although both the depression and the schizophrenia reviews show that intervention effects were sustained over greater than a 6- or 12-month period respectively, the precision of this estimate is reduced by the smaller number of trials included in this meta-analysis. Also, the measures of social functioning used by the trials may have affected the results of the review. Most of the scales used by the included trials do not include the full range of social functioning domains listed in online Table DS2, in particular parental functioning. Assessing the impact that depression has on parental roles among women is important ${ }^{46}$ as not only are they at a higher risk for depression, ${ }^{5}$ but maternal depression has been shown to affect child health ${ }^{47}$ and growth. ${ }^{48}$ Parental functioning was only measured in two of the ten scales used by the included trials, leading to potential underestimates of the effect of the intervention on social functioning in these trials. Furthermore, few of the tools to measure social functioning were developed or validated for the setting in which they were used, with some exceptions ${ }^{18}$ and there is a risk that contextually relevant outcomes, which may have the biggest impact on reducing stigma ${ }^{49}$ were not captured. Lastly, no trials were found that evaluated a number of types of psychosocial interventions shown to be effective in high-income countries, such as wellness promotion, ${ }^{50}$ vocational rehabilitation ${ }^{51}$ and cognitive remediation. ${ }^{52,53}$ Trials in LAMI countries evaluating the effect of these interventions on social functioning outcomes are needed.

On the other hand, the methods used for this review were strong. We used a wide-ranging search strategy with no limitations set on date, publication type or language. This resulted in the identification of a substantial body of previously largely uncited work from China that significantly adds to the body of knowledge particularly on the effectiveness of schizophrenia interventions. We conducted a meta-analysis of similar trials, using outcomes measured at similar time points and with comparable control groups to test the size of the effect of the interventions on social functioning, and examined heterogeneity by study quality.

\section{Implications}

The results of this review have a number of implications for future research.

(a) All trials of interventions for mental disorders in LAMI countries should use locally validated social functioning scales to measure social functioning outcomes in addition to measuring clinical and economic outcomes.

(b) Trial participants should be followed up for a sufficiently long time to detect changes in social functioning compared with clinical symptoms. Minimum follow-up times of 6 months for depression and 12 months for schizophrenia are recommended.

(c) Trials (particularly for schizophrenia) should be conducted of psychosocial interventions by non-specialist health workers, to directly inform efforts to scale up mental health services.

(d) Trials are needed of other psychosocial interventions such as wellness promotion, vocational rehabilitation and cognitive remediation, which hold promise for delivering improvements in social functioning but which have not yet been evaluated in LAMI countries.

Developing interventions that improve social functioning is important for a number of reasons. First, there is increasing evidence that service users place greater value on improvements in social functioning than improvements in clinical status ${ }^{54-56}$ and that impairments in social functioning are often a key factor in an individual's decision to seek care. ${ }^{3}$ Second, it has been suggested that seeing individuals with mental disorders successfully treated and return to socially productive roles has the greatest impact on reducing stigma ${ }^{57}$ and may succeed where concerted efforts at improving mental health literacy have failed. ${ }^{58}$ Ultimately, social functioning is seen as an increasingly important factor for reducing the overall burden of mental disorders, particularly for chronic or recurrent conditions such as schizophrenia and depression that cause very high levels of disability. ${ }^{59}$

This review provides strong evidence for depression and weaker evidence for schizophrenia in support of the use of a range of psychosocial interventions, with or without concurrent pharmacological interventions $s^{5,6}$ in LAMI countries. Many of the interventions included in the review were delivered by nonspecialists in collaborative and/or stepped-care delivery models often in primary care or community settings. The scarcity of specialist human resources in these settings ${ }^{60}$ indicates that these packages of care should be delivered by non-specialists working under the supervision of specialists, who provide capacitybuilding, continued supervision and referral pathways to enhance the effectiveness of these interventions. ${ }^{61}$ These findings therefore 
directly inform efforts such as the World Health Organization (WHO) Mental Health Gap Action Programme ${ }^{56}$ to scale up mental health services in LAMI countries. This review also supports calls to monitor the social functioning of patients as part of routine clinical practice ${ }^{3,46}$ in order to ensure that treatments go beyond clinical effectiveness and meet the wider needs of patients. Providing interventions that improve patient social functioning will not only reduce the burden of mental disorders by enabling people to fulfil a productive social role, but may also be the most effective way to combat stigma.

Mary J. De Silva, BA, MSC, PhD, Centre for Global Mental Health, London School of Hygiene and Tropical Medicine, London, UK; Sara Cooper, MPH, Centre for Public Mental Health, University of Cape Town, Cape Town, South Africa; Henry Lishi Li, BA, MSc, Centre for Global Mental Health, London School of Hygiene and Tropical Medicine, London, UK; Crick Lund, MA, MSocSci (Clin Psych), PhD, Centre for Public Mental Health, University of Cape Town, Cape Town, South Africa; Vikram Patel, MSc MRCPsych PhD FmedSci, Centre for Global Mental Health, London School of Hygiene and Tropical Medicine, London, UK, and Sangath, Alto-Porvorim, Goa, India

Correspondence: Mary J. De Silva, BA, MSC, PhD, Centre for Global Mental Health, London School of Hygiene and Tropical Medicine, Keppel Street, London WC1E 7HT, UK. Email: mary.desilva@Ishtm.ac.uk

First received 23 May 2012, final revision 27 Nov 2012, accepted 4 Dec 2012

\section{Funding}

This paper was produced as part of the Programme for Improving Mental health carE (PRIME), funded by the UK Department for International Development (DfID) for the benefit of LAMI countries (HRPC10). V.P. is supported by a Wellcome Trust Senior Research of LAMl countries (HRPC10). V.
Fellowship in Tropical Medicine.

\section{Acknowledgements}

We are grateful to Karen Blackhall and Hugh McGuire (Cochrane information scientists) for assistance developing the search strategy and conducting the searches, to Phil Edwards for statistical advice on the meta-analysis, and to Mercedes Hernandez for help collating references.

\section{Appendix}

\section{Inclusion and exclusion criteria for review}

\section{Study population}

Included: study conducted in LAMI country as defined by the World Bank. Any age.

Excluded: study conducted in high-income country.

\section{Condition of interest}

Included: depressive disorders and schizophrenia.

Excluded: other mental disorders, for example substance misuse, bipolar and anxiety disorders.

\section{Intervention}

Included: any psychosocial intervention (non-pharmacological) aimed at improving the lives of people with mental health problems and their families. This includes psychotherapy, social and collaborative care interventions where a number of different interventions are combined. Excluded: interventions not administered to the person with the mental health problem or their families/carer (for example interventions for healthcare staff).

\section{Study design}

Included: individual and cluster RCTs. Effectiveness trials with a placebo or TAU control group.

Excluded: non-randomised intervention studies, case-control or crosssectional studies. Equivalence trials with an active control group.

\section{Outcome}

Included: social functioning measured using a validated tool.

Excluded: individual measures of social functioning such as marital status, employment status or quality of social relationships. Quality of life measures.

\section{Meta-analysis}

Included: study reports a quantitative estimate of the effect of the intervention on the outcome suitable for combination in a meta-analysis. Excluded: no quantitative estimate of effect suitable for combination in a meta-analysis.

\section{References}

1 World Health Organization. The Global Burden of Disease: 2004 Update. WHO, 2004.

2 Kohn R, Saxena S, Levav I, Saraceno B. The treatment gap in mental health care. Bull World Health Organ 2004; 82: 858-66.

3 Hirschfeld RM, Montgomery SA, Keller MB, Kasper S, Schatzberg AF, Moller $\mathrm{HJ}$, et al. Social functioning in depression: a review. J Clin Psychiatry 2000; 61: $268-75$.

4 Patel V, Thornicroft G. Packages of care for mental, neurological, and substance use disorders in low- and middle-income countries: PLOS medicine series. PloS Med 2009; 6: e1000160.

5 Patel V, Simon G, Chowdhary N, Kaaya S, Araya R. Packages of care for depression in low- and middle-income countries. PLOS Med 2009; 6 : e1000159.

6 Mari J, Razzouk D, Thara R, Eaton J, Thornicroft G. Packages of care for schizophrenia in low- and middle-income countries. PLOS Med 2009; 6 : e1000165.

7 Dua T, Barbui C, Clark N, Fleischmann A, Poznyak V, van Ommeren M, et al. Evidence-based guidelines for mental, neurological, and substance use disorders in low- and middle-income countries: summary of WHO recommendations. PLoS Med 2011; 8: e1001122.

8 Moher D, Liberati A, Tetzlaff J, Altman DG. Preferred reporting items for systematic reviews and meta-analyses: the PRISMA statement. PLOS Med 2009; 6: e1000097.

9 Dagnan D. Psychosocial interventions for intellectual disabilities. In Psychiatric and Behavioural Disorders in Intellectual and Developmental Disabilities (eds N Bouras, G Holt): 330-8. Cambridge University Press, 2007

10 Murray C, Lopez AD. The Global Burden Disease: A Comprehensive Assessment of Mortality and Disability From Disease, Injuries and Risk Factors in 1990 and Projected to 2020. Harvard University Press, 1998.

11 Wiersma D. Measuring social disabilities in mental health. Soc Psychiatry Psychiatr Epidemiol 1996; 31: 101-8.

12 Mueser KT, Tarrier N. Handbook of Social Functioning in Schizophrenia. Allyn \& Bacon, 1998

13 Ware JE, Sherbourne CD. THE MOS 36-item short-form health survey (SF-36).1. Conceptual framework and item selection. Med Care 1992; 30 473-83.

14 Higgins JPT, Green S. Cochrane Handbook for Systematic Reviews of Interventions Version 5.1.0. The Cochrane Collaboration, 2011.

15 Gates S. Methodological guidelines. In About The Cochrane Collaboration (Collaborative Review Groups (CRGs)), issue 2 (eds The Editorial team: Pregnancy and Childbirth Group). Cochrane Collaboration, 2005.

16 Burns T, Patrick D. Social functioning as an outcome measure in schizophrenia studies. Acta Psychiatr Scand 2007; 116: 403-18.

17 Bolton P, Bass J, Neugebauer R, Verdeli H, Clougherty KF, Wickramaratne P, et al. Group interpersonal psychotherapy for depression in rural Uganda: a randomized controlled trial. JAMA 2003; 289: 3117-24.

18 Bass J, Neugebauer R, Clougherty KF, Verdeli H, Wickramaratne P, Ndogoni L, et al. Group interpersonal psychotherapy for depression in rural Uganda: 6-month outcomes. Randomised controlled trial. Br J Psychiatry 2006; 188 $567-73$.

19 de Mello MF, Myczcowisk LM, Menezes PR. A randomized controlled trial comparing moclobemide and moclobemide plus interpersonal psychotherapy in the treatment of dysthymic disorder. J Psychother Pract Res 2001; 10: $117-23$

20 Ye HP, Ming L. Group interpersonal psychotherapy for inpatient with major depression [in Chinese]. Chin Ment Health J 2006; 20: 524-6.

21 Patel V, Chisholm D, Rabe-Hesketh S, Dias-Saxena F, Andrew G, Mann A. Efficacy and cost-effectiveness of drug and psychological treatments for 
common mental disorders in general health care in Goa, India: a randomised, controlled trial. Lancet 2003; 361: 33-9.

22 Wei Q. Effect of new Morita therapy plus antipsychotic drugs in ameliorating post-schizophrenia depression [in Chinese]. Zhongguo Linchuang Kangfu 2005; 9: 72-5.

23 Patel V, Weiss HA, Chowdhary N, Naik S, Pednekar S, Chatterjee S, et al. Lay health worker led intervention for depressive and anxiety disorders in India: impact on clinical and disability outcomes over 12 months. Br J Psychiatry 2011; 199: 459-66.

24 Araya R, Rojas G, Fritsch R, Gaete J, Rojas M, Simon G, et al. Treating depression in primary care in low-income women in Santiago, Chile: a randomised controlled trial. Lancet 2003; 361: 995-1000.

25 Rojas G, Fritsch R, Solis J, Jadresic E, Castillo C, González M, et al. Treatment of postnatal depression in low-income mothers in primary-care clinics in Santiago, Chile: a randomised controlled trial. Lancet 2007; 370: 1629-37.

26 Vitriol VG, Ballesteros ST, Florenzano RU, Weil KP, Benadof DF. Evaluation of an outpatient intervention for women with severe depression and a history of childhood trauma. Psychiatr Serv 2009; 60: 936-42.

27 Fritsch R, Araya R, Solís J, Montt E, Pilowsky D, Rojas G. A randomized tria of pharmacotherapy with telephone monitoring to improve treatment of depression in primary care in Santiago, Chile [in Spanish]. Rev Med Chile 2007; 135: 587-95.

28 Hu X, Wang YL, Fu HP. Synthetical family treatment for depression: a randomized-controlled single-blind study among 76 cases [in Chinese] J Clin Rehabil Tissue Eng Res 2007; 11: 7787-90.

29 Cohen J. Statistical Power Analysis in the Behavioral Sciences (2nd edn). Lawrence Erlbaum Associates, 1988.

30 Xiang M, Ran M, Li S. A controlled evaluation of psychoeducational family intervention in a rural Chinese community. Br J Psychiatry 1994; 165: 544-8.

31 Cui Y, Yang W, Weng Y. Effectiveness of social skills training in patients with chronic schizophrenia [in Chinese]. Chin Ment Health J 2004; 18: 799-805.

32 Xiong W, Phillips MR, Hu X, Wang R, Dai Q, Kleinman J, et al. Family-based intervention for schizophrenic patients in China. A randomised controlled trial. Br J Psychiatry 1994; 165: 239-47.

33 Li Z, Arthur D. Family education for people with schizophrenia in Beijing China. Randomised controlled trial. Br J Psychiatry 2005; 187: 339-45.

34 Wang NS, Li RQ, Zhan LY, Wang GS, Liu L. The effect of regular health education on rehabilitation of outpatients with schizophrenia in rural areas [in Chinese]. Chin Ment Health J 2008; 22: 709-12.

35 Wei X, Ma S, Hua F, Xu D. Primary application of mental education in recovery stage of schizophrenia [in Chinese]. Chin Ment Health J 1997; 11: 186-7.

36 Chen $\mathrm{H}$, Chang $\mathrm{Y}$, Xiang $\mathrm{Y}$. Efficacy of the rehabilitation strategy for inpatients with schizophrenia [in Chinese]. Chin Ment Health J 2003; 17: 839-42

37 Guo X, Zhai J, Liu Z, Fang M, Wang B, Wang C, et al. Effect of antipsychotic medication alone vs combined with psychosocial intervention on outcomes of early-stage schizophrenia. Arch Gen Psychiatry 2010; 67: 893-904.

38 Yildiz M, Veznedaroglu B, Eryavuz A, Kayahan B. Psychosocial skills training on social functioning and quality of life in the treatment of schizophrenia: a controlled study in Turkey. Int J Psychiatry Clin Pract 2004; 8: 219-25.

39 Zimmer $\mathrm{M}$, Duncan AV, Laitano $\mathrm{D}$, Ferreira $\mathrm{EE}$, Belmonte-de-Abreu $\mathrm{P}$. A twelve-week randomized controlled study of the cognitive-behaviora integrated psychological therapy program: positive effect on the social functioning of schizophrenic patients. Rev Bras Psiquiatr 2007; 29: 140-7.

40 Meng P, Zheng R, Cai Z, Cao D, Ma L, Lui J, et al. Group intervention for schizophrenia inpatient with art as medium [in Chinese]. Acta Psychol Sinica 2005; 37: 403-12.
41 Li X, Li X, Ma X, Ni Y. Effects of social rehabilitation on late-onse schizophrenia [in Chinese]. Chin Ment Health J 2002; 16: 711-3.

42 Pang $Y$, Huang S, Li X, Hu L, Lin C. The effect of health education on compliance of patients with paranoid schizophrenia in their follow-up therapy [in Chinese]. Chin Ment Health J 2002; 16: 348-50.

43 Sterne JAC, Harbord RM. Funnel plots in meta-analysis. Stata J 2004; 4: 127-41.

44 Schulz KF, Chalmers I, Hayes RJ, Altman DG. Empirical evidence of bias. Dimensions of methodological quality associated with estimates of treatment effects in controlled trials. JAMA 1995; 273: 408-12.

45 Chisholm D, Flisher AJ, Lund C, Patel V, Saxena S, Thornicroft G, et al. Scale up services for mental disorders: a call for action. Lancet 2007; 370 1241-52.

46 Weissman MM. Social functioning and the treatment of depression. J Clin Psychiatry 2000; 61 (suppl 1): 33-8.

47 Rahman A, Malik A, Sikander S, Roberts C, Creed F. Cognitive behaviour therapy-based intervention by community health workers for mothers with depression and their infants in rural Pakistan: a cluster-randomised controlled trial. Lancet 2008; 372: 902-9.

48 Surkan PJ, Kennedy CE, Hurley KM, Black MM. Maternal depression and early childhood growth in developing countries: systematic review and meta-analysis. Bull World Health Organ 2011; 89: 608-15.

49 Patel V, Farooq S, Thara R. What is the best approach to treating schizophrenia in developing countries? PLOS Med 2007; 4: e159.

50 Chafetz L, White M, Collins-Bride G, Cooper BA, Nickens J. Clinical trial of wellness training: health promotion for severely mentally ill adults. J Nerv Ment Dis 2008; 196: 475-83.

51 Marshall M, Crowther R, Almaraz-Serrano A, Creed F, Sledge W, Kluiter H, et al. Systematic reviews of the effectiveness of day care for people with severe mental disorders: (1) acute day hospital versus admission; (2) vocational rehabilitation; (3) day hospital versus outpatient care. Health Technol Assess 2001; 5: 1-75.

52 Nuechterlein KH, Dawson ME, Ventura J, Gitlin M, Subotnik KL, Snyder KS, et al. The vulnerability/stress model of schizophrenic relapse: a longitudinal study. Acta Psychiatr Scand Suppl 1994; 382: 58-64.

53 Delahunty A, Morice R. Rehabilitation of frontal/executive impairments in schizophrenia. Aust N Z J Psychiatry 1996; 30: 760-7.

54 Read UM, Adiibokah E, Nyame S. Local suffering and the global discourse of mental health and human rights: an ethnographic study of responses to mental illness in rural Ghana. Glob Health 2009; 5: 13.

55 Davidson L, White $\mathrm{W}$. The concept of recovery as an organising principle for integrating mental health and addiction services. J Behav Health Serv Res 2007; 34: 104-20.

56 Mental Health Gap Action Programme. Scaling Up Care for Mental, Neurological, and Substance Use Disorders. World Health Organization, 2008.

57 Yang L, Lo G, WonPat-Borja A, Singla D, Link B, Phillips M. Effects of labeling and interpersonal contact upon attitudes towards schizophrenia: implications for reducing mental illness stigma in urban China. Soc Psychiatry Psychiatr Epidemiol 2012; 47: 1459-73.

58 Schomerus G, Schwahn C, Holzinger A, Corrigan P, Grabe H, Carta M, et al. Evolution of public attitudes about mental illness: a systematic review and meta-analysis. Acta Psychiatr Scand 2012; 125: 440-52.

59 Lopez D, Mathers D, Ezzati M, Jamison T, Murray J. Global Burden of Disease and Risk Factors. Oxford University Press and The World Bank, 2006.

60 Kakuma R, Minas H, van Ginneken N, Dal Poz MR, Desiraju K, Morris JE, et al. Human resources for mental health care: current situation and strategies for action. Lancet 2011; 378: 1654-63.

61 Patel V. The future of psychiatry in low- and middle-income countries. Psychol Med 2009; 39: 1759-62. 